\title{
BREAKING WAVE DETECTION WITH THE PHASE-TIME METHOD (PTM) REVISITED
}

\author{
Stefan Schimmels, Forschungszentrum Küste (FZK), Hannover, Germany, schimmels@fzk-nth.de \\ Dan Liberzon, Technion-Israel Institute of Technology, Haifa, Israel, liberzon@technion.sc.il
}

\section{INTRODUCTION}

Breaking waves are a very complicated phenomenon associated with high energy transfer rates and turbulence levels and can create tremendous loads when impacting on a structure. Therefore an exact understanding of the wave breaking process is of major interest in coastal and offshore engineering research. In this context the point of breaking inception is of critical importance, e.g. for breaking wave impact studies, but despite lots of research efforts still no objective and unique criterion exists and the breaking point needs to be estimated by tedious and subjective analysis of video data. The Phase-Time Method (PTM), as introduced by Huang et al. (1992), was shown to be able to overcome these discrepancies and to provide an objective method for the determination of the point of breaking inception as demonstrated by Zimmermann and Seymour (2002) and Irschik et al. (2010). However, their threshold based approaches still seem to be slightly ambiguous and lack general validity. In the present work the PTM and the suggested breaking detection methods will be reviewed and thoroughly evaluated. The most critical issues will be addressed and new approaches to interpret the PTM data will be proposed.

\section{METHODOLOGY, RESULTS AND DISCUSSION}

The PTM is based on the Hilbert transformation, which allows transforming a time signal into time varying amplitudes and frequencies. Based on the observation that wave breaking is associated with a significant increase of the instantaneous frequency, Zimmerman and Seymour (2002) suggested for irregular deep water waves that breaking starts when the deviation from a mean frequency, which they obtained from the de-trended phase function, exceeds a threshold. The ambiguity in this approach is reflected by the fact that Zimmermann and Seymour (2002) suggested a threshold value of about $0.5 \mathrm{~Hz}$, while Irschik et al. (2011) found values between 0.6 and $0.7 \mathrm{~Hz}$ for focused waves. For the latter Fig. 1 shows exemplarily the water surface elevation and instantaneous frequency at three gauges, before, at and after breaking inception. The sudden frequency increase and the exceedance of the threshold value shown by the dashed line can be clearly observed. Based on this and other examples from laboratory experiments new approaches will be presented, which consider different criteria like shape and pattern of the frequency function and the signals at neighboring gauges. The double peak after breaking inception in Fig. 1 was also observed by Zimmermann and Seymour (2002) and is one example for an additional breaking criterion. The new approaches shall reduce ambiguity in the threshold based method in order to provide a unique and objective method, which will help to further improve our understanding of braking wave physics.
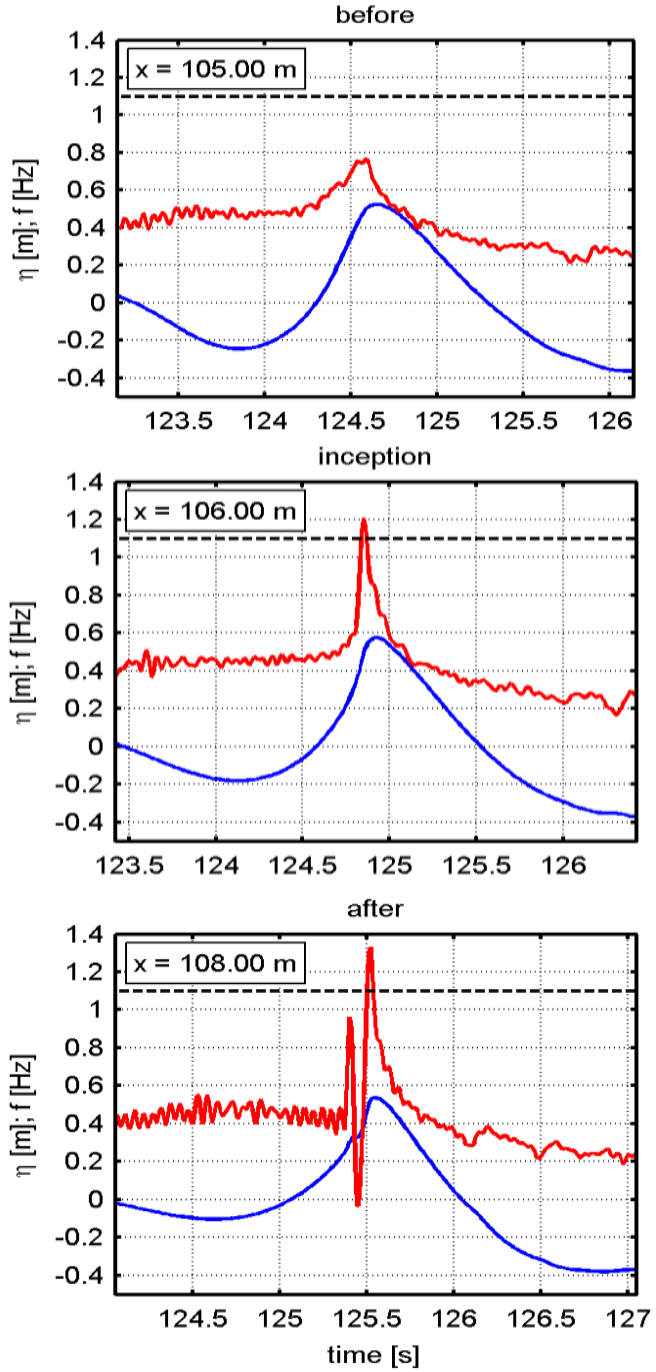

Figure 1. Evolution of water surface elevation (blue) and instantaneous frequency (red) of a breaking focused wave.

\section{REFERENCES}

Huang, N. E., Long, S. R., Tung, C. C., Donelan, M. A., Yuan, Y., Lai, R. J., 1992. The local properties of ocean waves by the phasetime method, Geophysical Research Letters, Vol. 19, Issue 7, 685-688.

Irschik, K., Schimmels, S., Oumeraci, H., 2011. Breaking criteria for laboratory experiments based on the PhaseTime Method (PTM), Coastal Engineering Proceedings, 1(32), waves.6.

Zimmermann, C.-A., Seymour, R., 2002. Detection of breaking in a deep water wave record. Journal of Waterway, Port, Coastal, and Ocean Engineering, Vol. 128, Issue 2, 72-78. 